\title{
Article
}

\section{Prognostic Value of the Neutrophil-to-Lymphocyte Ratio before and after Radiotherapy for Anaplastic Thyroid Carcinoma}

\author{
Jiyun Park ${ }^{1,+}$, Jun Park ${ }^{1,+} \oplus$, Jung-Hee Shin ${ }^{2}$, Young-Lyun $\mathrm{Oh}^{3}{ }^{3}$, Hyun-Ae Jung ${ }^{4}$, Man-Ki Chung ${ }^{5}$, Jun-Ho Choe ${ }^{6}$, \\ Yong-Chan Ahn ${ }^{7} \mathbb{(}$, Sun-Wook Kim ${ }^{1}$, Jae-Hoon Chung ${ }^{1}{ }^{1}$, Tae-Hyuk Kim ${ }^{1, * \mathbb{D}}$ and Jae-Myoung Noh $^{7, *}$
}

1 Division of Endocrinology and Metabolism, Department of Medicine, Samsung Medical Center, Sungkyunkwan University School of Medicine, Seoul 06351, Korea; nove1123.park@samsung.com (J.P.); pjun113@gmail.com (J.P.); swkimmd@skku.edu (S.-W.K.); thyroid@skku.edu (J.H.C.)

2 Department of Radiology and Center for Imaging Science, Thyroid Center, Samsung Medical Center, Sungkyunkwan University School of Medicine, Seoul 06351, Korea; helena35.shin@samsung.com

3 Department of Pathology, Thyroid Center, Samsung Medical Center, Sungkyunkwan University School of Medicine, Seoul 06351, Korea; yl.oh@samsung.com

4 Division of Hematology-Oncology, Department of Medicine, Samsung Medical Center, Sungkyunkwan University School of Medicine, Seoul 06351, Korea; hyunae.jung@samsung.com

5 Department of Otorhinolaryngology-Head and Neck Surgery, Samsung Medical Center, Sungkyunkwan University School of Medicine, Seoul 06351, Korea; manki.chung@samsung.com

6 Division of Breast and Endocrine Surgery, Department of Surgery, Samsung Medical Center, Sungkyunkwan University School of Medicine, Seoul 06351, Korea; junho.choe@samsung.com

7 Department of Radiation Oncology, Samsung Medical Center, Sungkyunkwan University School of Medicine, Seoul 06351, Korea; ycahn.ahn@samsung.com

check for updates

Citation: Park, J.; Park, J.; Shin, J.-H.; Oh, Y.-L.; Jung, H.-A.; Chung, M.-K.; Choe, J.-H.; Ahn, Y.-C.; Kim, S.-W.; Chung, J.-H.; et al. Prognostic Value of the Neutrophil-to-Lymphocyte Ratio before and after Radiotherapy for Anaplastic Thyroid Carcinoma. Cancers 2021, 13, 1913. https:// doi.org/10.3390/cancers13081913

Academic Editor: Barbara Jarzab

Received: 4 March 2021

Accepted: 13 April 2021

Published: 15 April 2021

Publisher's Note: MDPI stays neutral with regard to jurisdictional claims in published maps and institutional affiliations.

Copyright: (c) 2021 by the authors. Licensee MDPI, Basel, Switzerland. This article is an open access article distributed under the terms and conditions of the Creative Commons Attribution (CC BY) license (https:/ / creativecommons.org/licenses/by/ $4.0 /)$.
* Correspondence: taehyukmd.kim@samsung.com (T.-H.K.); rodrno@skku.edu (J.-M.N.); Tel.: +82-2-3410-6049 (T.-H.K.); +82-2-3410-2612 (J.-M.N.)

+ These two authors contributed equally to this work.

Simple Summary: Systemic hematologic markers such as the neutrophil-lymphocyte ratio (NLR) are attracting attention in a simple blood test predicting survival and treatment outcomes in various solid cancers. In our study including 40 patients with anaplastic thyroid cancer (ATC) who underwent radiotherapy, we found that high NLR before and after radiotherapy was associated with poor survival. This study result means that host immunity has an important role in patients with ATC, which can be important information for designing future investigations on the impact of immune system modulating therapy in ATC.

Abstract: The neutrophil-lymphocyte ratio (NLR) is a marker of systemic inflammation, and its elevation has recently been associated with poor survival in many solid cancers. Leukocyte elevation and lymphocyte reduction are associated with a poor response to radiotherapy (RT). This study aimed to assess the prognostic value of NLR before and after RT for anaplastic thyroid carcinoma (ATC). This retrospective study analyzed 40 patients with ATC who received RT with available complete blood cell count data from November 1995 through May 2020 at Samsung Medical Center (Seoul, Korea). Patients were classified into two groups according to the NLR before and after RT. The median overall survival (OS) was 8.9 months (range, 3.5-18.2) in the low NLR group $(<3.47)$ and 5.2 months (range, 2.7-7.5) months in the high NLR group ( $\geq 3.47$ ). The association between NLR and OS was also observed in multivariable Cox regression analysis (hazard ratio, 3.18; 95\% confidence interval, $1.15-8.85 ; p=0.026)$. The OS curves differed significantly according to post-RT NLR $(p=0.036)$. A high NLR before and after RT may be significantly associated with poor OS in patients with ATC who receive $\mathrm{RT}$.

Keywords: anaplastic thyroid carcinoma; neutrophil-to-lymphocyte ratio; neutrophil; lymphocyte; radiotherapy 


\section{Introduction}

Anaplastic thyroid carcinoma (ATC) is an aggressive form of thyroid cancer that is associated with a poor prognosis. The management of patients with ATC is very difficult, and there is no effective therapy; however, multimodal therapy (MMT) is commonly recommended [1-3].

Several studies have reported that systemic hematological markers can predict poor outcomes in some malignancies. In particular, the baseline neutrophil-to-lymphocyte ratio (NLR), which represents the ratio of peripheral circulating neutrophil and lymphocyte counts, is a marker of inflammation, and increased NLR has been shown to be an indicator of a poor prognosis in many cancers, including that of the breast, colon, and head and neck [4-6]. With regard to thyroid cancers, it is known that a high baseline NLR is associated with increased tumor size, metastasis, and disease-free survival in patients with differentiated thyroid cancer [7-9]. Moreover, NLR can be used to discriminate aggressive forms of thyroid cancer from well differentiated cancer [10].

Recently, not only the baseline NLR but also NLR before and after treatments such as chemoradiotherapy or immunotherapy was reported to be associated with survival in several solid tumors [4,11-14]. Leukocytosis is associated with resistance to radiotherapy (RT). On the other hand, lymphocytes are sensitive to RT and exhibit antitumor effects $[4,15]$. Furthermore, RT can lead to lymphopenia and reduced lymphocyte function [14]. Considering the interaction between changes in hematological values and RT, it can be hypothesized that NLR before and after RT can play a prognostic role in the evaluation of treatment outcomes in several cancers. However, to our knowledge, no previous studies have investigated the prognostic value of NLR before and after RT for ATC.

The primary aim of this study was to assess the prognostic impact of NLR before and after RT in patients with ATC.

\section{Materials and Methods}

\subsection{Study Population}

From November 1995 through May 2020, 120 patients diagnosed with ATC were treated at Samsung Medical Center, Seoul, Republic of Korea. Among these patients, 64 received RT. The exclusion criteria were as follows: previously differentiated carcinoma $(n=5)$, anaplastic change $<5 \%(n=1)$, unavailable baseline complete blood cell count $(\mathrm{CBC})$ and differential count data $(n=17)$, and palliative surgery after definitive concurrent chemoradiotherapy $(n=1)$. A total of 40 patients were included, 19 of whom underwent surgery and RT with or without systemic treatment such as tyrosine kinase inhibitor (TKI) therapy or cytotoxic chemotherapy. The other 21 patients received only RT with or without systemic treatment. Figure 1 shows the study flowchart. The study was approved by the Institutional Review Board of Samsung Medical Center (SMC-IRB 2020-10-033).

\subsection{Laboratory and Pathological Data}

Patient and tumor characteristics and $\mathrm{CBC}$ data were extracted from the electronic medical records. Only CBC data available for counting absolute lymphocytes and absolute neutrophils were used. NLR was calculated as the absolute neutrophil count (ANC) divided by the absolute lymphocyte count (ALC). In the group who underwent surgery, baseline CBC data were obtained on a date nearest to surgery date when the interval between surgery and RT was within 100 days. However, for one patient with an interval longer than 100 days, data were obtained on a date closest to the RT start date. In the group that did not undergo surgery, baseline $\mathrm{CBC}$ data were obtained on a date nearest to the RT start date. Post-RT CBC data were obtained within 4-6 weeks after the last day of RT. The American Joint Committee on Cancer (AJCC) staging system (8th edition) was used for tumor staging [16]. 


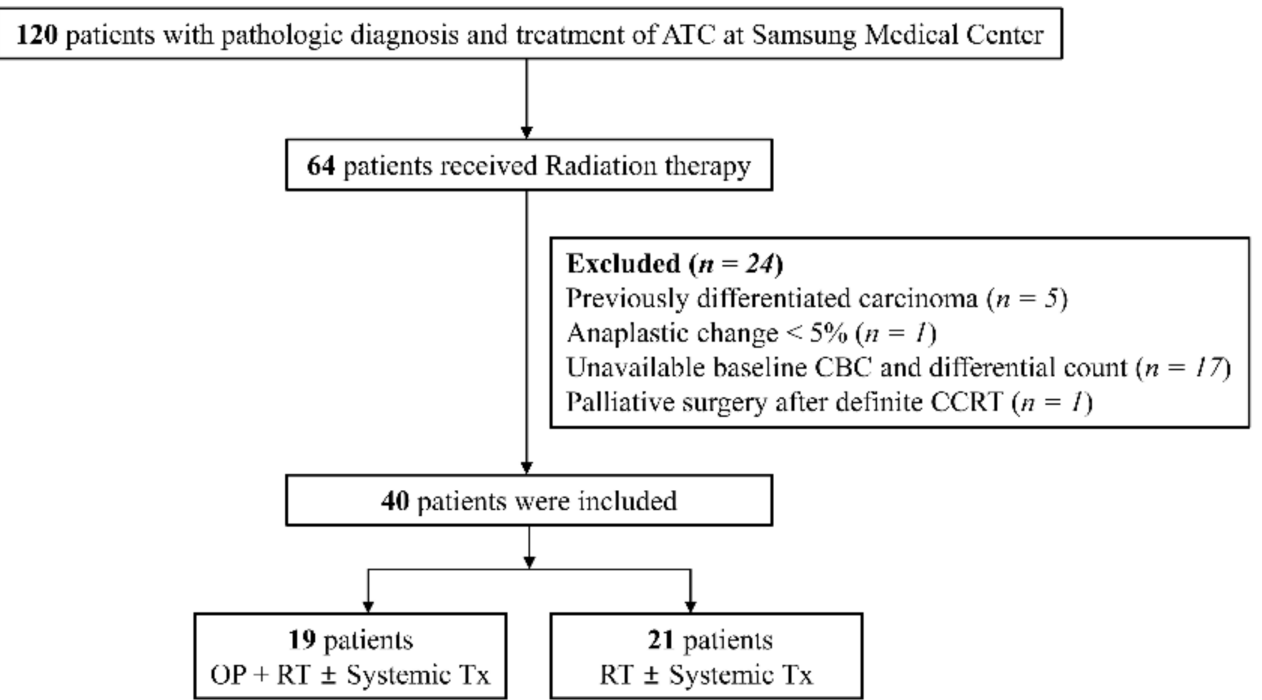

Figure 1. Study flowchart. ATC, anaplastic thyroid carcinoma; OP, operation; CBC, complete blood count; CCRT, concur rent chemoradiotherapy; Tx, treatment.

\subsection{Treatment and Primary Outcome}

Surgical interventions included total thyroidectomy with or without bilateral neck dissection as per the surgeon's decision. For cytotoxic chemotherapy, a regimen including doxorubicin, cisplatin, and paclitaxel was administered before surgery or as a form of concurrent chemoradiotherapy. For patients treated with TKIs, multikinase inhibitors such as lenvatinib and selective $B R A F$ inhibitors such as dabrafenib plus trametinib or vemurafenib were administered when the patients had BRAF600E mutation.

RT was delivered with 4-, 6-, or 10-MV photons generated from a linear accelerator. Thirteen (32.5\%) and $20(50.0 \%)$ patients received three-dimensional conformal RT (3DCRT) and intensity-modulated RT (IMRT), respectively. The remaining seven (17.5) patients received two-dimensional RT, and most of them were treated before 2002. For 3D-CRT and IMRT, the gross tumor volume (GTV) was defined as the volume of the primary tumor and involved lymph node(s) on the basis of all available clinical information. The clinical target volume (CTV) was delineated by adding 3 $-5 \mathrm{~mm}$ margins in all directions from the GTV, and the margins were optionally modified in accordance with the anatomical boundaries. In the case of postoperative RT, the CTV included the primary tumor bed and pathologically involved regional lymphatics with adequate margins. The radiation dose fractionation was based on the aim of RT and the patient's performance status. For example, $30 \mathrm{~Gy}$ in 10 fractions was prescribed for eight patients requiring palliative RT, while 59.4 Gy in 27 fractions was prescribed for eight patients requiring postoperative RT.

MMT was defined as a combination of two or more of the following treatments: surgery, cytotoxic chemotherapy, TKI therapy, and RT. The primary outcome was overall survival (OS), which was calculated as the time from the date of diagnosis to the occurrence of death from any cause.

\subsection{Statistical Analysis}

All data are presented as medians with interquartile ranges (IQRs) for continuous variables and numbers with percentages for categorical variables. The t-test and Wilcoxon rank-sum test were used to compare continuous variables depending on whether a normal distribution was satisfied. The chi-square test was used for categorical variables. Area under the curve (AUC) value using receiver operating characteristic (ROC) analysis was performed to evaluate the optimal cutoff value for NLR. Kaplan-Meier analysis and the log-rank test were used to compare survival between the two groups. Hazard ratios (HRs) and $95 \%$ confidence intervals (CIs) for OS were calculated by univariate and multivariate analyses using the multivariable Cox proportional hazard regression method. Variables 
with a $p$-value $\leq 0.2$ in univariate analysis were included in multivariate analysis. All $p$-values were two-tailed, and $p$-values of $<0.05$ were considered statistically significant. All statistical analyses were performed using STATA version 16.0 (StataCorp, College Station, TX, USA).

\section{Results}

\subsection{Patient Characteristics}

Table 1 shows the baseline characteristics according to the use of surgical resection. The median age at diagnosis was 65.1 years (IQR 57.8-72.2) in the surgery group and 70.8 years (IQR 58.1-78.6) in the non-surgery group. The median tumor size was $4.8 \mathrm{~cm}$ (IQR 3.1-5.4) in the surgery group and $5.3 \mathrm{~cm}$ (IQR 4.2-6.5) $\mathrm{cm}$ in the non-surgery group. The stage IVC disease was higher in the group without surgery than in the group with surgery $(71.4 \%$ vs. $31.6 \%, p=0.012)$, while the patients who received a total RT dose of EQD2 10 (equivalent dose in 2-Gy fractions with an $\alpha / \beta$ ratio of 10 ) of 60 Gy or more was significantly higher in the group with surgery $(n=12(63.1 \%)$ with surgery vs. $n=2$ $(9.5 \%)$ without surgery, $p=0.012)$. The frequency of systemic treatment, including cytotoxic chemotherapy and TKI therapy, was not different between the two groups $(p=0.273$ and $p$ $=0.201$, respectively). There were significantly more patients with an NLR of $\geq 3.47$ in the group without surgery than in the group with surgery $(n=12(57.1 \%)$ without surgery vs. $n=3(15.8 \%)$ with surgery, $p=0.007)$.

Table 1. Baseline characteristics of patients who received RT with or without surgery for anaplastic thyroid carcinoma.

\begin{tabular}{|c|c|c|c|}
\hline Variables & $\begin{array}{c}\mathrm{OP}+\mathrm{RT} \pm \text { Systemic Tx } \\
(n=19)\end{array}$ & $\begin{array}{c}\mathrm{RT} \pm \underset{(n=21)}{\text { Systemic } \mathrm{Tx}} \\
\end{array}$ & $p$-Value \\
\hline Age (years) & $65.1(57.8-72.2)$ & $70.8(58.1-78.6)$ & 0.383 \\
\hline Sex & & & 0.220 \\
\hline Male & $9(47.4)$ & $6(28.6)$ & - \\
\hline Female & $10(52.6)$ & $15(71.4)$ & - \\
\hline Tumor size $(\mathrm{cm})$ & $4.8(3.1-5.4)$ & $5.3(4.2-6.5)$ & 0.245 \\
\hline Stage & & & 0.012 \\
\hline IVA \& IVB & $13(68.4)$ & $6(28.6)$ & - \\
\hline IVC & $6(31.6)$ & $15(71.4)$ & \\
\hline Total dose (EQD2 10, Gy) & & & $<0.001$ \\
\hline$<60.0$ & $7(36.9)$ & $19(90.5)$ & - \\
\hline$\geq 60.0$ & $12(63.1)$ & $2(9.5)$ & - \\
\hline Systemic treatment & & & - \\
\hline Cytotoxic chemotherapy & $5(26.32)$ & $9(42.86)$ & 0.273 \\
\hline TKI & $3(15.8)$ & $7(33.3)$ & 0.201 \\
\hline Multimodal therapy & $19(100)$ & $13(61.9)$ & 0.003 \\
\hline Baseline ALC $(\mu \mathrm{L})$ & $2120(1740-2363)$ & $1810(1310-2380)$ & 0.173 \\
\hline Baseline ANC $(\mu \mathrm{L})$ & $4220(2970-5803)$ & $6598(5814-11,729)$ & 0.017 \\
\hline Baseline NLR & $2.24(1.4-3.1)$ & $4.95(2.54-6.60)$ & 0.013 \\
\hline Baseline NLR $(\geq 3.47)$ & $3(15.8)$ & $12(57.1)$ & 0.007 \\
\hline
\end{tabular}

Continuous variables are presented as medians (interquartile ranges). Categorical variables are presented as numbers (percentages). ALC, absolute lymphocyte count; ANC, absolute leukocyte count; EQD2 10 , equivalent dose in 2-Gy fractions with an $\alpha / \beta$ ratio of 10; NLR, neutrophil-to-lymphocyte ratio; RT, radiotherapy; OP, operation; TKI, tyrosine kinase inhibitor; $\mathrm{Tx}$, treatment.

\subsection{Optimal Cutoff Value for the NLR and Cinicopathological Characteristics According to NLR}

The median and IQR of NLR in the total population were 2.87 (1.50-5.28). To evaluate the optimal cutoff value for NLR, the ROC curve for OS was used. The optimal cutoff value for the baseline NLR was set to 3.47, with an area under the curve (AUC) of 0.64 (sensitivity 48.4, specificity 88.9, Figure S1). The optimal cutoff value for NLR after RT was 3.44, with an AUC of 0.65 (sensitivity 76.5, specificity 62.5, Figure S2). According to these results, the 
patients were classified into two groups (high NLR group, NLR $\geq 3.47, n=15$ (37.5\%); low NLR group, NLR <3.47, $n=25(62.5 \%))$.

The clinicopathological characteristics of patients according to NLR are shown in Table 2. The NLR was 1.99 (1.28-2.67) in the low NLR group and 6.33 (4.59-11.11) in the high LNR group $(p<0.001)$. The tumor size and stage IVC disease were not different between the two groups $(4.5 \mathrm{~cm}$ (IQR 3.3-5.5) in the low NLR group vs. $5.4 \mathrm{~cm}$ (IQR 4.4-6.5) in the high NLR group, $p=0.145 ; n=11(44 \%)$ in the low NLR group vs. $n=10(66.7 \%)$ in the high NLR group, $p=0.165)$. Surgical resection was higher in the low NLR group $(64.0 \%$ vs. $20.0 \%, p=0.007$ ).

Table 2. Clinicopathological characteristics according to the baseline NLR in patients with anaplastic thyroid carcinoma treated by radiotherapy.

\begin{tabular}{|c|c|c|c|}
\hline Variables & $\begin{array}{l}\text { Low NLR } \\
(n=25)\end{array}$ & $\begin{array}{c}\text { High NLR } \\
(n=15)\end{array}$ & $p$-Value \\
\hline Age (years) & $67.4(63.1-72.2)$ & $67.3(57.9-73.0)$ & 0.882 \\
\hline Sex & & & 0.354 \\
\hline Male & $8(32.0)$ & $7(53.3)$ & - \\
\hline Female & $17(68.0)$ & $8(46.7)$ & - \\
\hline Tumor size $(\mathrm{cm})$ & $4.5(3.3-5.5)$ & $5.4(4.4-6.5)$ & 0.145 \\
\hline Stage & & & 0.165 \\
\hline IVA \& IVB & $14(56.0)$ & $5(33.3)$ & - \\
\hline IVC & $11(44.0)$ & $10(66.7)$ & - \\
\hline Total dose (EQD2 $10, \mathrm{~Gy})$ & & & 0.123 \\
\hline$<60.0$ & $14(56.0)$ & $12(80.0)$ & - \\
\hline$\geq 60.0$ & $11(44.0)$ & $3(20.0)$ & - \\
\hline Surgery & $16(64.0)$ & $3(20.0)$ & 0.007 \\
\hline Systemic treatment & & & \\
\hline Cytotoxic chemotherapy & $7(28.0)$ & $7(46.7)$ & 0.231 \\
\hline TKI & $6(24.0)$ & $4(26.7)$ & 0.850 \\
\hline Multimodal therapy & $20(80.0)$ & $12(80.0)$ & 1.000 \\
\hline Baseline NLR & $1.99(1.28-2.67)$ & $6.33(4.95-11.11)$ & $<0.001$ \\
\hline Baseline ALC $(\mu \mathrm{L})$ & $2218(1890-2460)$ & $1480(1222-1853)$ & $<0.001$ \\
\hline Baseline ANC $(\mu \mathrm{L})$ & $4188(2680-5802)$ & $10,494(7677-14,060)$ & $<0.001$ \\
\hline
\end{tabular}

$\overline{\text { Continuous variables are presented as medians (interquartile ranges). Categorical variables are presented as }}$ numbers (percentages). ALC, absolute lymphocyte count; ANC, absolute leukocyte count; EQD210, equivalent dose in 2-Gy fractions with an $\alpha / \beta$ ratio of 10; NLR, neutrophil-to-lymphocyte ratio; TKI, tyrosine kinase inhibitor.

\subsection{Overall Survival According to the Baseline NLR}

Figure 2 shows the Kaplan-Meier curves for OS. The high NLR was associated with poorer survival $(p=0.002)$. The median OS was 8.9 months (IQR 3.5-18.2) in the low NLR group and 5.2 months (IQR 2.7-7.5) in the high NLR group. Stage IVC disease, surgery, MMT, a high total radiation dose, and a high NLR were associated with OS in univariate Cox regression analysis (Table 3). However, only high NLR was associated with OS in multivariate Cox regression analysis for the total population (HR: 3.18, 95\% CI: $1.15-8.85$, $p=0.026$; Table 3).

\subsection{Subgroup Analysis in the Group with and without Surgery}

Figure 3 shows the Kaplan-Meier curves for OS in the surgery and non-surgery groups ( $p=0.017$ vs. $p=0.376$, respectively). A high NLR was associated with poor survival in the group with surgery, but not in the group without surgery. In univariate Cox regression analysis for the surgery group, a high total radiation dose and high NLR were associated with OS. However, in multivariate analysis, only a high total radiation dose remained a significant factor (high total radiation dose, HR: 0.05, 95\% CI: 0.005-0.43, $p=0.007$; NLR, HR: 2.07, 95\% CI: $0.28-15.39, p=0.478$; Table 4 ). In the group without surgery, stage IVC and TKI therapy were associated with OS in univariate Cox analysis, and both of them 
remained as significant factors in multivariate Cox analysis (Stage IVC, HR: 9.37, 95\% CI: 2.22-39.46, $p=0.002$; TKI, HR: 0.14, 95\% CI: 0.04-0.51, $p=0.003$; Table 4).

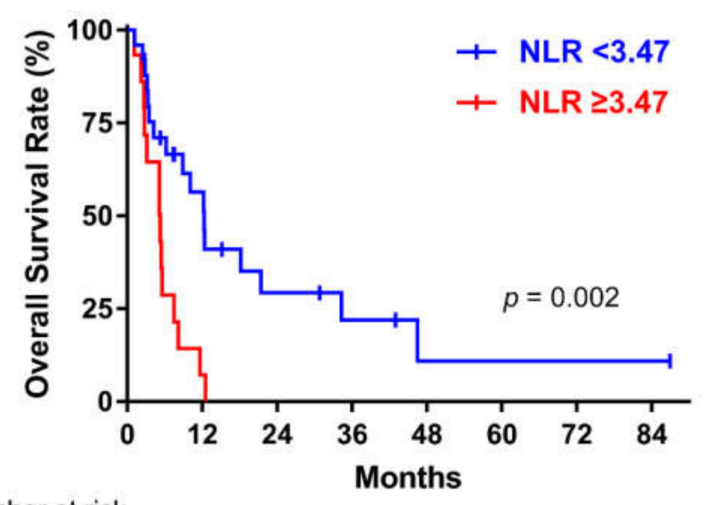

Number at risk

NLR $<3.47 \quad 25$

NLR $\geq 3.47 \quad 15$

$\begin{array}{lll}5 & 1 & 1 \\ 0 & 0 & 0\end{array}$

Figure 2. Overall survival according to the baseline neutrophil-to-lymphocyte ratio (NLR) in the overall sample of patients who underwent radiotherapy for anaplastic thyroid carcinoma.

Table 3. Cox proportional hazard model for all-cause mortality in the overall sample of patients with anaplastic thyroid carcinoma treated by radiotherapy.

\begin{tabular}{ccccc}
\hline \multirow{2}{*}{ Variables } & \multicolumn{2}{c}{ Univariate Analysis } & \multicolumn{2}{c}{ Multivariate Analysis } \\
\cline { 2 - 5 } & HR (95\% CI) & $p$-Value & HR (95\% CI) & $p$-Value \\
\hline Age (years) & $1.00(0.97-1.04)$ & 0.819 & - & - \\
Sex (male) & $1.18(0.56-2.48)$ & 0.661 & - & - \\
Size (cm) & $1.23(0.99-1.52)$ & 0.059 & $0.86(0.64-1.16)$ & 0.328 \\
Stage (IVC) & $3.59(1.59-8.07)$ & 0.002 & $2.97(0.81-10.95)$ & 0.102 \\
Surgery & $0.21(0.09-0.50)$ & $<0.001$ & $0.70(0.24-2.03)$ & 0.507 \\
Systemic treatment & & & & \\
Cytotoxic CTx & $1.57(0.74-3.33)$ & 0.238 & - & - \\
TKI & $0.76(0.33-1.72)$ & 0.506 & - & 0.236 \\
Multimodal therapy & $0.36(0.16-0.84)$ & 0.018 & $0.42(0.10-1.77)$ & 0.089 \\
EQD2 $10(\geq 60$ Gy) & $0.10(0.03-0.31)$ & $<0.001$ & $0.27(0.06-1.22)$ & 0.026 \\
NLR $(\geq 3.47)$ & $3.32(1.51-7.29)$ & 0.003 & $3.18(1.15-8.85)$ &
\end{tabular}

CI, confidence interval; CTx, chemotherapy; EQD2 10 , equivalent dose in 2-Gy fractions with an $\alpha / \beta$ ratio of 10; HR, hazard ratio; NLR, neutrophil-to-lymphocyte ratio; TKI, tyrosine kinase inhibitor.
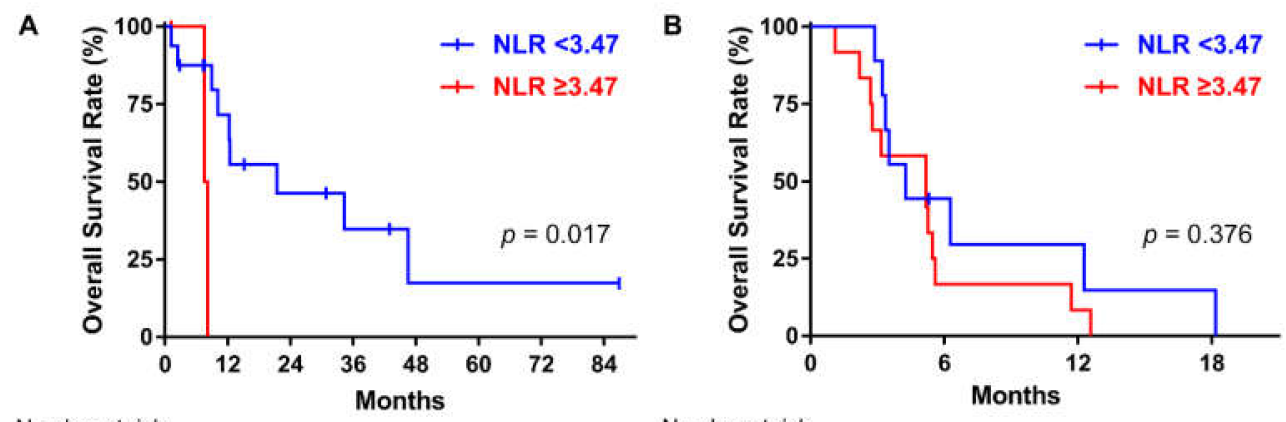

Number at risk
NLR $<3.47 \quad 16$

NLR $\geq 3.47 \quad 3$

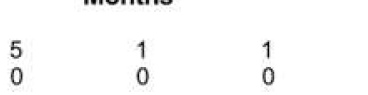

Number at risk
NLR $<3.47 \quad 9$

NLR $\geq 3.47 \quad 12$

Figure 3. Overall survival according to the neutrophil-to-lymphocyte ratio (NLR) in patients who underwent radiotherapy (RT) with or without surgery for anaplastic thyroid carcinoma. (A) Operation $+\mathrm{RT} \pm$ systemic treatment. (B) $\mathrm{RT} \pm$ systemic treatment. 
Table 4. Cox proportional hazard model for all-cause mortality in patients who received RT with or without surgery for anaplastic thyroid carcinoma.

\begin{tabular}{|c|c|c|c|c|c|c|c|c|}
\hline \multirow{3}{*}{$\begin{array}{l}\text { Treatment } \\
\text { Variables }\end{array}$} & \multicolumn{4}{|c|}{$\mathrm{OP}+\mathrm{RT} \pm$ Systemic Tx $(n=19)$} & \multicolumn{4}{|c|}{$\mathrm{RT} \pm$ Systemic Tx $(n=21)$} \\
\hline & \multicolumn{2}{|c|}{ Univariate Analysis } & \multicolumn{2}{|c|}{ Multivariate Analysis } & \multicolumn{2}{|c|}{ Univariate Analysis } & \multicolumn{2}{|c|}{ Multivariate Analysis } \\
\hline & $\begin{array}{c}\text { HR } \\
(95 \% \text { CI) }\end{array}$ & $p$-Value & $\begin{array}{c}\text { HR } \\
(95 \% \text { CI) }\end{array}$ & $p$-Value & $\begin{array}{c}\text { HR } \\
(95 \% \text { CI) }\end{array}$ & $p$-Value & $\begin{array}{c}\text { HR } \\
(95 \% \mathrm{CI})\end{array}$ & $p$-Value \\
\hline Age (years) & $\begin{array}{c}1.00 \\
(0.93-1.09)\end{array}$ & 0.831 & - & - & $\begin{array}{c}0.98 \\
(0.94-1.02)\end{array}$ & 0.375 & - & - \\
\hline Sex (male) & $\begin{array}{c}1.74 \\
(0.52-5.77)\end{array}$ & 0.369 & - & - & $\begin{array}{c}1.79 \\
(0.60-5.39)\end{array}$ & 0.298 & - & - \\
\hline Size $(\mathrm{cm})$ & $\begin{array}{c}1.03 \\
(0.67-1.58)\end{array}$ & 0.893 & - & - & $\begin{array}{c}1.14 \\
(0.88-1.46)\end{array}$ & 0.322 & - & - \\
\hline Stage (IVC) & $\begin{array}{c}2.21 \\
(0.52-9.37)\end{array}$ & 0.281 & - & - & $\begin{array}{c}2.96 \\
(0.95-9.22)\end{array}$ & 0.061 & $\begin{array}{c}9.37 \\
(2.22-39.46)\end{array}$ & 0.002 \\
\hline Systemic Tx & - & - & - & - & $\begin{array}{c}0.88 \\
(0.34-2.25)\end{array}$ & 0.787 & - & - \\
\hline Cytotoxic CTx & $\begin{array}{c}1.11 \\
(0.29-4.30)\end{array}$ & 0.883 & - & - & $\begin{array}{c}1.86 \\
(0.66-5.19)\end{array}$ & 0.238 & - & - \\
\hline TKI & $\begin{array}{c}0.74 \\
(0.15-3.52)\end{array}$ & 0.702 & - & - & $\begin{array}{c}0.44 \\
(0.16-1.17)\end{array}$ & 0.099 & $\begin{array}{c}0.14 \\
(0.04-0.51)\end{array}$ & 0.003 \\
\hline $\begin{array}{l}\text { EQD2 } 10 \\
(\geq 60 \text { Gy) }\end{array}$ & $\begin{array}{c}0.04 \\
(0.005-0.36)\end{array}$ & 0.004 & $\begin{array}{c}0.05 \\
(0.005-0.43)\end{array}$ & 0.007 & $\begin{array}{c}0.46 \\
(0.10-2.04)\end{array}$ & 0.304 & - & - \\
\hline NLR $(\geq 3.47)$ & $\begin{array}{c}7.76 \\
(1.08-55.92)\end{array}$ & 0.042 & $\begin{array}{c}2.07 \\
(0.28-15.39)\end{array}$ & 0.478 & $\begin{array}{c}1.52 \\
(0.59-3.90)\end{array}$ & 0.382 & - & - \\
\hline
\end{tabular}

CI, confidence interval; CTx, chemotherapy; EQD2 10 , equivalent dose in 2-Gy fractions with an $\alpha / \beta$ ratio of 10; HR, hazard ratio; NLR, neutrophil-to-lymphocyte ratio; OP, operation; RT, radiotherapy; TKI, tyrosine kinase inhibitor; Tx, treatment.

\subsection{Association between NLR after RT and OS}

In total, 25 patients with available CBC data after RT were included in this analysis. The median and IQR of post-RT-NLR were 6.60 (2.75-9.81). Ten patients had low post-RT NLR, and 15 patients had high post-RT NLR. Figure 4 shows the Kaplan-Meier curves for OS according to the post-RT-NLR. High post-RT-NLR was associated with poor OS $(p=0.036)$. The median OS was 13.9 (IQR, 3.5-34.3) and 4.3 (IQR, 2.5-7.5) months in the low and high post-RT-NLR groups, respectively. In univariate Cox analysis, the HR $(95 \%$ CI) for high post-RT-NLR was $2.98(1.03-8.68)(p=0.045)$.
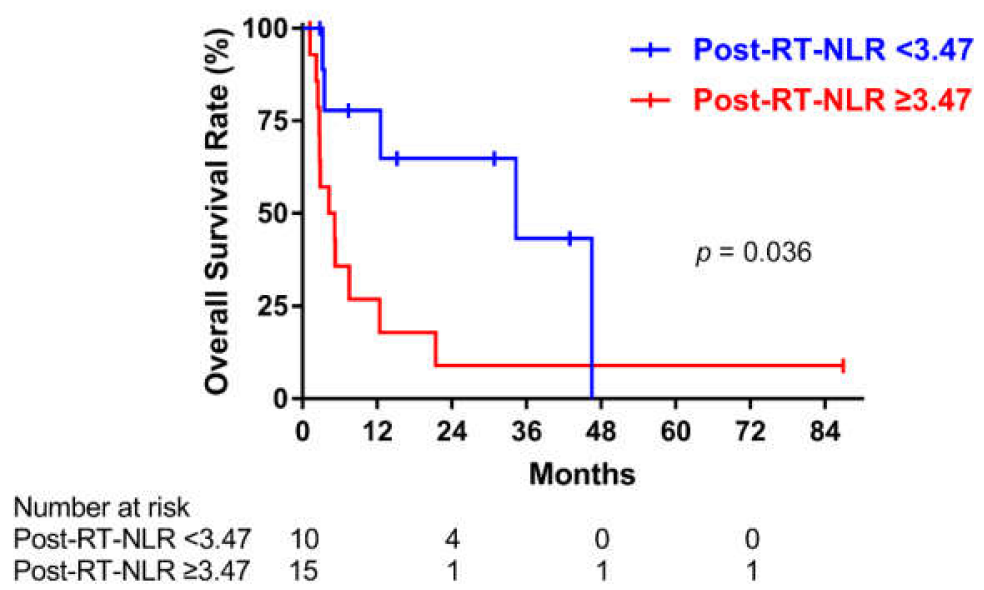

Figure 4. Overall survival according to the post-radiation therapy (post-RT) neutrophil-tolymphocyte ratio (NLR) in patients with anaplastic thyroid carcinoma.

When 25 patients were divided into four groups according to the change in NLR after RT (low-low, low-high, high-low, and high-high), there were no statistically significant 
differences in OS among the four groups ( $p=0.051$; Figure 5). However, the HR (95\% CI) for the high-high NLR group versus the low-low NLR group was $6.40(1.55-26.40)$ in univariate Cox analysis $(p=0.01)$.

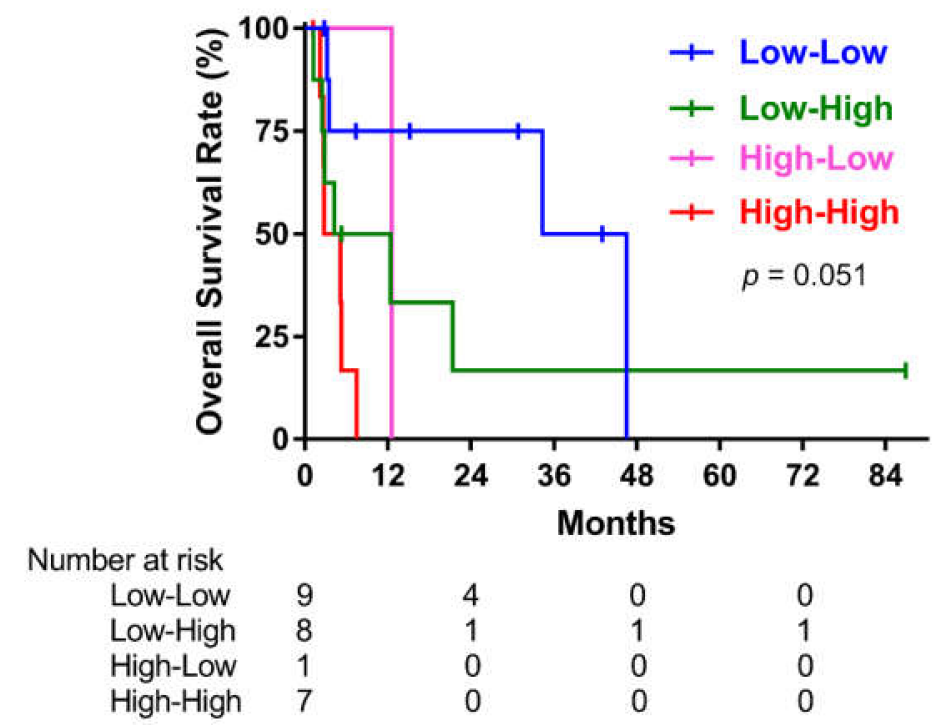

Figure 5. Overall survival according to the change in the neutrophil-to-lymphocyte ratio (NLR) after radiotherapy for anaplastic thyroid carcinoma.

\section{Discussion}

In this study, we evaluated whether NLR before and after RT for ATC was associated with OS. The high baseline NLR was significantly associated with poorer OS in both univariate and multivariate analyses for the total population. The subgroup analysis showed that the high NLR group had poor OS in the patients who underwent surgery. In this subgroup with surgery, high NLR lost its significant association with OS in multivariate Cox analysis; however, the high total radiation dose remained significant. Because only three patients had high NLR, we could not conduct further stratified analysis for evaluating the interaction between NLR and the radiation dose. The high post-RT NLR was also associated with poor OS. Only 25 out of 40 patients had available CBC data after RT, and 14 patients of them underwent surgery while 11 did not undergo surgery. A univariate Cox analysis was attempted to confirm the association between RT dose and OS in patients with post-RT-NLR value, but this was not possible because the number of surviving patients in the group with a total radiation dose of $<60$ Gy was quite small (Table S1).

Although high NLR has been reported to be associated with poor survival in many solid cancers, the association between ATC and NLR has not been reported. The role of the inflammatory cells in the development and progression of thyroid cancer has been discussed in previous studies [17-19]. To date, it is known that chronic lymphocytic thyroiditis in PTC induced improved prognosis, with fewer lymphocytes in patients with poorly differentiated thyroid cancer (PDTC) and ATC than in PTC patients, suggesting lymphocytes have a protective role in thyroid cancer [18]. Monocytes accelerate tumor progression and suppress normal immune responses, and a low lymphocyte-to-monocyte ratio is associated with poor survival in ATC $[15,17]$. Neutrophils secrete highly reactive metabolites and eliminate pathogens. However, these metabolites can induce mutation or proliferation of the cancer cells [18]. Our study showed that high NLR was associated with poor survival before and after RT in ATC, which is consistent with results in previous studies considering the role of each inflammatory cell.

As we mentioned in the introduction, it was reported that high NLR was associated with increased tumor size, metastasis, and disease-free survival in patients with differentiated thyroid cancer $[7,8]$. As the tumor size and staging increase, higher amounts of 
tumor-derived or tumor-induced factors are likely to be released into the periphery of cancer cells, thus increasing the amount of circulating neutrophils [20]. In our study, tumor size was not associated with NLR. PTC is an indolent form of cancer that grows slowly, and the NLR value is also lower compared to ATC [10]. This means inflammation may be less important in the initiation of carcinogenesis in differentiated cancer, with a lower effect on systemic inflammation [8]. The NLR value may increase as the size increases, gradually provoking systemic inflammation in PTC. On the other hand, ATC is aggressive and grows fast, inducing inflammatory processes in the early phase of carcinogenesis. Therefore, NLR was thought not to be associated with tumor size. Furthermore, one study reported that NLR can discriminate aggressive forms of thyroid cancer from well differentiated cancer [10]. In this previous study, the NLR of PTC, with a median value of 1.57 (range, 0.28-16.29), could discriminate PDTC and ATC from PTC, and the cut-off value of LNR for discriminating PDTC from ATC was 3.8. The NLR of our study population was 2.87 (range, 0.94-22.51), a higher value compared to PTC patients in a previous study. However, only 14 out of 40 in our study population had NLR values equal to or higher than 3.8 . When the cut off value was set to 3.8 , the sensitivity was $45.16 \%$ and specificity was $89 \%$ for death in our study population. This means that even in ATC patients, NLR greater than 3.8 represents high mortality. Considering PDTC is as aggressive as ATC, LNR greater than 3.8 can discriminate aggressive types of thyroid cancer and poor survival. Studies on larger patient cohorts are warranted to define a uniform NLR cut-off value for thyroid cancer.

High NLR is also associated with poorer outcomes in patients with immune checkpoint inhibitors in other solid cancers [21]. Circulating neutrophil counts are associated with tumor infiltrating neutrophils [22]. Therefore, decreased circulating lymphocytes are associated with reduced lymphocyte in tumor tissue, resulting in less chance to interact with immune check-point inhibitors. Further intervention studies are needed to confirm that NLR can predict the response of immunotherapy in thyroid cancer.

Although our study population included patients who received RT, surgery, MMT, and a high total radiation dose were associated with better survival, as reported in previous studies [3,23]. In particular, our subgroup analysis showed that the total radiation dose was a good prognostic factor for OS in patients who received surgery, while TKI treatment was a good prognostic factor for patients who received RT without surgery. This means that a high-dose adjuvant RT for patients receiving surgery and adjuvant TKI therapy for patients receiving RT without surgery could improve OS in patients with ATC. A previous study reported that a high radiation dose resulted in better survival, even in patients with unresected ATC [24]. In our study population, more than $90 \%$ of the patients without surgery received low-dose RT. Therefore, there was a limitation in evaluating the association between the radiation dose and OS in the group without surgery.

Several studies have reported that selective BRAF inhibitors or multikinase inhibitors are effective in patients with ATC who have previously undergone surgery or RT $[25,26]$. In our study, only three patients in the surgery group received TKI treatment, and TKI treatment was not associated with OS in this group. However, in the group without surgery, TKI treatment was performed in more than thirty percent of the patients and was also associated with better OS, as shown in previous studies [24,25]. Further prospective studies are warranted to validate this association.

This study has some limitations. First, it was a small, single-center, retrospective study; therefore, there are some inherent limitations such as selection bias and validation. Second, CBC data were not available for all patients, and patients' medical condition or situation at the time of CBC measurement was not known. NLR is a readily available and affordable biomarker; however, it can be affected by unmeasurable medical conditions; therefore, NLR values have to be interpreted cautiously in a clinical context. Third, we could not perform additional immunohistochemical staining to find tumor-associated neutrophils or flow cytometry to evaluate the lymphocyte subtype due to a lack of available tissue specimens. Therefore, the difference in neutrophil and lymphocyte infiltration in ATC between low NLR and high NLR groups was not evaluated. However, we focused on the 
association between blood NLR and OS of the patients with ATC based on the assumption that blood NLR could reflect tumor tissue NLR. Further studies are needed to confirm the consistency of NLR in blood and tissue.

\section{Conclusions}

High NLR was associated with poor survival in patients with ATC who received RT. Furthermore, high post-RT NLR was also associated with poor survival. This study evaluated the role of NLR as a readily available and affordable blood marker for predicting survival in ATC patients before and after RT. Prospective studies are needed to verify the association between NLR and OS before and after RT in patients with ATC.

Supplementary Materials: The following are available online at https:/ / www.mdpi.com/article/10 .3390 / cancers13081913/s1, Table S1: Overall survival according to the total radiation dose (based on $60 \mathrm{~Gy}$ ) in patients with available complete blood cell count data after radiotherapy for anaplastic thyroid carcinoma, Figure S1: ROC curve assessing the cutoff points of baseline NLR, Figure S2: ROC curve assessing the cutoff points of post-RT-NLR.

Author Contributions: Conceptualization, J.P. (Jiyun Park), T.-H.K. and J.-M.N.; methodology, J.P. (Jiyun Park), J.P. (Jun Park), T.-H.K. and J.-M.N.; formal analysis, J.P. (Jiyun Park); investigation, J.P. (Jiyun Park), J.P. (Jun Park) and H.-A.J.; writing—original draft, J.P. (Jiyun Park), T.-H.K. and J.-M.N.; writing-review and editing, J.P. (Jiyun Park), T.-H.K. and J.-M.N.; supervision, J.-H.S., Y.-L.O., H.-A.J., M.-K.C., J.-H.C., Y.-C.A., S.-W.K. and J.-H.C. All authors have read and agreed to the published version of the manuscript.

Funding: This research received no external funding.

Institutional Review Board Statement: This study complied with the ethical standards of the Helsinki Declaration and was approved by the Institutional Review Board of Samsung Medical Center (SMC-IRB 2020-10-033).

Informed Consent Statement: Not applicable.

Data Availability Statement: The data presented in this study are available within the article and Supplementary Material.

Conflicts of Interest: The authors declare no conflict of interest.

\section{References}

1. Pezzi, T.A.; Mohamed, A.S.R.; Sheu, T.; Blanchard, P.; Sandulache, V.C.; Lai, S.Y.; Cabanillas, M.E.; Williams, M.D.; Pezzi, C.M.; $\mathrm{Lu}, \mathrm{C}$; ; et al. Radiation therapy dose is associated with improved survival for unresected anaplastic thyroid carcinoma: Outcomes from the National Cancer Data Base. Cancer 2017, 123, 1653-1661. [CrossRef]

2. Park, J.W.; Choi, S.H.; Yoon, H.I.; Lee, J.; Kim, T.H.; Kim, J.W.; Lee, I.J. Treatment outcomes of radiotherapy for anaplastic thyroid cancer. Radiat. Oncol. J. 2018, 36, 103-113. [CrossRef]

3. Fan, D.; Ma, J.; Bell, A.C.; Groen, A.H.; Olsen, K.S.; Lok, B.H.; Leeman, J.E.; Anderson, E.; Riaz, N.; McBride, S.; et al. Outcomes of multimodal therapy in a large series of patients with anaplastic thyroid cancer. Cancer 2020, 126, 444-452. [CrossRef]

4. Moon, H.; Roh, J.-L.; Lee, S.-W.; Kim, S.-B.; Choi, S.-H.; Nam, S.Y.; Kim, S.Y. Prognostic value of nutritional and hematologic markers in head and neck squamous cell carcinoma treated by chemoradiotherapy. Radiother. Oncol. 2016, 118, 330-334. [CrossRef]

5. Azab, B.; Mohammad, F.; Shah, N.; Vonfrolio, S.; Lu, W.; Kedia, S.; Bloom, S.W. The value of the pretreatment neutrophil lymphocyte ratio vs. platelet lymphocyte ratio in predicting the long-term survival in colorectal cancer. Cancer Biomark. 2014, 14, 303-312. [CrossRef] [PubMed]

6. Azab, B.; Shah, N.; Radbel, J.; Tan, P.; Bhatt, V.; Vonfrolio, S.; Habeshy, A.; Picon, A.; Bloom, S. Pretreatment neutrophil/lymphocyte ratio is superior to platelet/lymphocyte ratio as a predictor of long-term mortality in breast cancer patients. Med. Oncol. 2013, 30, 1-11. [CrossRef] [PubMed]

7. Liu, C.-L.; Lee, J.-J.; Liu, T.-P.; Chang, Y.-C.; Hsu, Y.-C.; Cheng, S.-P. Blood neutrophil-to-lymphocyte ratio correlates with tumor size in patients with differentiated thyroid cancer. J. Surg. Oncol. 2013, 107, 493-497. [CrossRef] [PubMed]

8. Manatakis, D.K.; Tseleni-Balafouta, S.; Balalis, D.; Soulou, V.N.; Korkolis, D.P.; Sakorafas, G.H.; Plataniotis, G.; Gontikakis, E. Association of Baseline Neutrophil-to-Lymphocyte Ratio with Clinicopathological Characteristics of Papillary Thyroid Carcinoma. Int. J. Endocrinol. 2017, 2017, 1-7. [CrossRef]

9. Feng, J.; Wang, Y.; Shan, G.; Gao, L. Clinical and prognostic value of neutrophil-lymphocyte ratio for patients with thyroid cancer. Med. 2020, 99, e19686. [CrossRef] 
10. Cho, J.-S.; Park, M.-H.; Ryu, Y.-J.; Yoon, J.-H. The neutrophil to lymphocyte ratio can discriminate anaplastic thyroid cancer against poorly or well differentiated cancer. Ann. Surg. Treat. Res. 2015, 88, 187-192. [CrossRef] [PubMed]

11. Liu, J.; Ma, F.; Sun, B.; Cong, Y.; Xuan, L.; Wang, Q.; Wu, S. Predictive Value of Lymphocyte-Related Blood Parameters at the Time Point of Lymphocyte Nadir During Radiotherapy in Breast Cancer. OncoTargets Ther. 2020, ume 13, 151-161. [CrossRef]

12. Jensen, G.L.; Blanchard, P.; Gunn, G.B.; Garden, A.S.; Fuller, C.D.; Sturgis, E.M.; Gillison, M.L.; Phan, J.; Morrison, W.H.; Rosenthal, D.I.; et al. Prognostic impact of leukocyte counts before and during radiotherapy for oropharyngeal cancer. Clin. Transl. Radiat. Oncol. 2017, 7, 28-35. [CrossRef]

13. Lalani, A.-K.A.; Xie, W.; Martini, D.J.; Steinharter, J.A.; Norton, C.K.; Krajewski, K.M.; Duquette, A.; Bossé, D.; Bellmunt, J.; Van Allen, E.M.; et al. Change in neutrophil-to-lymphocyte ratio (NLR) in response to immune checkpoint blockade for metastatic renal cell carcinoma. J. Immunother. Cancer 2018, 6, 5. [CrossRef] [PubMed]

14. Yoon, C.I.; Kim, D.; Ahn, S.G.; Bae, S.J.; Cha, C.; Park, S.; Park, S.; Kim, S.I.; Lee, H.S.; Park, J.Y.; et al. Radiotherapy-Induced High Neutrophil-to-Lymphocyte Ratio is a Negative Prognostic Factor in Patients with Breast Cancer. Cancers 2020, $12,1896$. [CrossRef] [PubMed]

15. Ahn, J.; Song, E.; Oh, H.-S.; Song, D.E.; Kim, W.B.; Kim, T.Y.; Shong, Y.K.; Jeon, M.J. Low Lymphocyte-to-Monocyte Ratios Are Associated with Poor Overall Survival in Anaplastic Thyroid Carcinoma Patients. Thyroid 2019, 29, 824-829. [CrossRef] [PubMed]

16. Perrier, N.D.; Brierley, J.D.; Tuttle, R.M. Differentiated and anaplastic thyroid carcinoma: Major changes in the American Joint Committee on Cancer eighth edition cancer staging manual. CA: A Cancer J. Clin. 2017, 68, 55-63. [CrossRef] [PubMed]

17. French, J.D.; Bible, K.; Spitzweg, C.; Haugen, B.R.; Ryder, M. Leveraging the immune system to treat advanced thyroid cancers Lancet Diabetes Endocrinol. 2017, 5, 469-481. [CrossRef]

18. Guarino, V.; Castellone, M.D.; Avilla, E.; Melillo, R.M. Thyroid cancer and inflammation. Mol. Cell. Endocrinol. 2010, 321, 94-102. [CrossRef] [PubMed]

19. Ugolini, C.; Basolo, F.; Proietti, A.; Vitti, P.; Elisei, R.; Miccoli, P.; Toniolo, A. Lymphocyte and Immature Dendritic Cell Infiltrates in Differentiated, Poorly Differentiated, and Undifferentiated Thyroid Carcinoma. Thyroid 2007, 17, 389-393. [CrossRef]

20. Moses, K.; Brandau, S. Human neutrophils: Their role in cancer and relation to myeloid-derived suppressor cells. Semin. Immunol. 2016, 28, 187-196. [CrossRef]

21. Sacdalan, D.B.; Lucero, J.A.; Sacdalan, D.L. Prognostic utility of baseline neutrophil-to-lymphocyte ratio in patients receiving immune checkpoint inhibitors: A review and meta-analysis. OncoTargets Ther. 2018, ume 11, 955-965. [CrossRef]

22. Takakura, K.; Ito, Z.; Suka, M.; Kanai, T.; Matsumoto, Y.; Odahara, S.; Matsudaira, H.; Haruki, K.; Fujiwara, Y.; Saito, R.; et al. Comprehensive assessment of the prognosis of pancreatic cancer: Peripheral blood neutrophil-lymphocyte ratio and immunohistochemical analyses of the tumour site. Scand. J. Gastroenterol. 2016, 51, 610-617. [CrossRef] [PubMed]

23. Swaak-Kragten, A.T.; De Wilt, J.H.; Schmitz, P.I.; Bontenbal, M.; Levendag, P.C. Multimodality treatment for anaplastic thyroid carcinoma-Treatment outcome in 75 patients. Radiother. Oncol. 2009, 92, 100-104. [CrossRef] [PubMed]

24. Saini, S.; Tulla, K.; Maker, A.V.; Burman, K.D.; Prabhakar, B.S. Therapeutic advances in anaplastic thyroid cancer: A current perspective. Mol. Cancer 2018, 17, 1-14. [CrossRef]

25. Subbiah, V.; Kreitman, R.J.; Wainberg, Z.A.; Cho, J.Y.; Schellens, J.H.; Soria, J.C.; Wen, P.Y.; Zielinski, C.; Cabanillas, M.E.; Urbanowitz, G.; et al. Dabrafenib and Trametinib Treatment in Patients With Locally Advanced or Metastatic BRAF V600-Mutant Anaplastic Thyroid Cancer. J. Clin. Oncol. 2018, 36, 7-13. [CrossRef] [PubMed]

26. Iyer, P.C.; Dadu, R.; Ferrarotto, R.; Busaidy, N.L.; Habra, M.A.; Zafereo, M.; Gross, N.; Hess, K.R.; Gule-Monroe, M.; Williams, M.D.; et al. Real-World Experience with Targeted Therapy for the Treatment of Anaplastic Thyroid Carcinoma. Thyroid 2018, 28, 79-87. [CrossRef] [PubMed] 